$\frac{12}{8}-2495850$

UCRL-ID-119155

\title{
Observation of Spectral Broadening in a Commercial Modelocked and Q-Switched Nd:YLF Oscillator- Wegner's Demon
}

\author{
P. Wegner \\ M. Feit
}

September 14, 1994

This is an informal report intended primarily for internal or limited external distribution. The opinions and conchusions stated are those of the author and may or may not be those of the Laboratory.

Work performed under the auspices of the U.S. Department of Energy by the Lwrence Livermore National Laboretory under Contract W-7405-ENG-18. 


\section{DISCLAIMER}

This document was prepared as an account of work sponsored by an agency of the United States Government. Neither the United States Government nor the University of California nor any of their employees, makes any warranty, express or implied, or assumes any legal liability or responsibility for the accuracy, completeness, or usefulness of any information, apparatus, product, or process disclosed, or represents that its use would not infringe privately owned rights. Reference herein to any specific commercial product, process, or service by trade name, trademark, manufacturer, or otherwise, does not necessarily constitute or imply its endorsement, recommendation, or favoring by the United States Government or the University of California. The views and opinions of authors expressed herein do not necessarily state or reflect those of the United States Government or the University of California, and shall not be used for advertising or product endorsement purposes.

This report has been reproduced directly from the best available copy.

Available to DOE and DOE contractors from the Office of Scientific and Technical Information P.O. Box 62, Oak Ridge, TN 37831

Prices available from (615) 576-8401, FTS 626-8401

Available to the public from the

National Technical Information Service

U.S. Department of Commerce

5285 Port Royal Rd.,

Springfield, VA 22161 


\section{DISCLAIMER}

Portions of this document may be illegible in electronic image products. Images are produced from the best available original document. 
To: Distribution

From: P. Wegner and M. Feit

Subject: Observation of spectral broadening in a commercial modelocked and Q-switched Nd:YLF oscillator- Wegner's Demon (see Figure)

\section{Summary}

We have observed spectral broadening in the pulsed output of a Quantronix model 4217 modelocked and Q-switched Nd:YLF oscillator. When Q-switched, the 76-MHz oscillator produces a nearly gaussian train of pulses with a total energy of $3 \mathrm{~mJ}$ and a duration of $\sim 300 \mathrm{~ns}$. Between $Q-$ switch cycles the CW-pumped oscillator lases at a low level to enable a circulating modelocked pulse to reach steady-state transform-limited conditions. With a 1-mm intracavity etalon the steady-state modelocked pulsewidth is $150 \mathrm{ps}$ and the oscillating bandwidth is $3 \mathrm{GHz}$. Measurements show that at the peak of the Q-switched train the modelocked pulse bandwidth is broadened to $11 \mathrm{GHz}$.

We attribute the altered spectrum to self-phase modulation (SPM) of the circulating pulse in the 3.8-cm long acousto-optic quartz modulators. SPM has been recognized as a potential problem in this type of oscillator however we did not expect to find it in a commercial product and its occurrence in the modulators rather than the rod was a surprise. Analysis of the cavity mode has determined that the spot size is small at the ends of the cavity where the modulators are located. The peak intensities achieved at these locations during Q-switching are estimated to exceed 4 $\mathrm{GW} / \mathrm{cm}^{2}$ and the peak roundtrip B-integral is estimated at $\sim .75$ radians. A large mode volume in the rod coupled with a relatively small nonlinear coefficient makes intensity-dependent phase shifts there negligible by comparison.

Since the effects of SPM in this type of oscillator are not well-documented we developed a numerical model and used it to examine the spectral evolution of the circulating modelocked pulse after Q-switching (see Figure 1). The model incorporates a rod with gain, two passive quartz blocks with nonlinear refractive index for simulating nonlinear propagation in the modulators, a time-dependent shutter to simulate the active modelocker, an etalon, and distributed Fresnel losses. The spectra that we calculate are in good agreement with that observed, confirming the source of the broadening. The oscillator has been redesigned to increase the mode size in the modulators and eliminate the SPM.

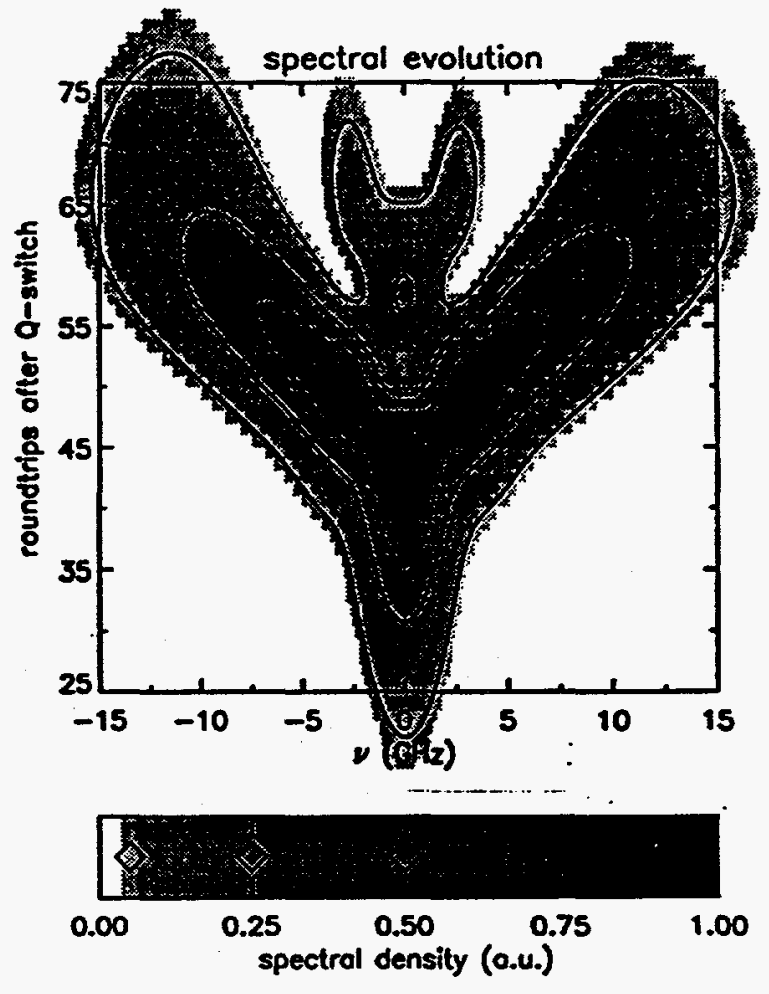

Figure 1. Calculation showing how the spectrum of the circulating modelocked pulse in the oscillator evolves after the cavity is Q-switched. Maximum energy occurs on the $53^{\text {rd }}$ roundtrip. 


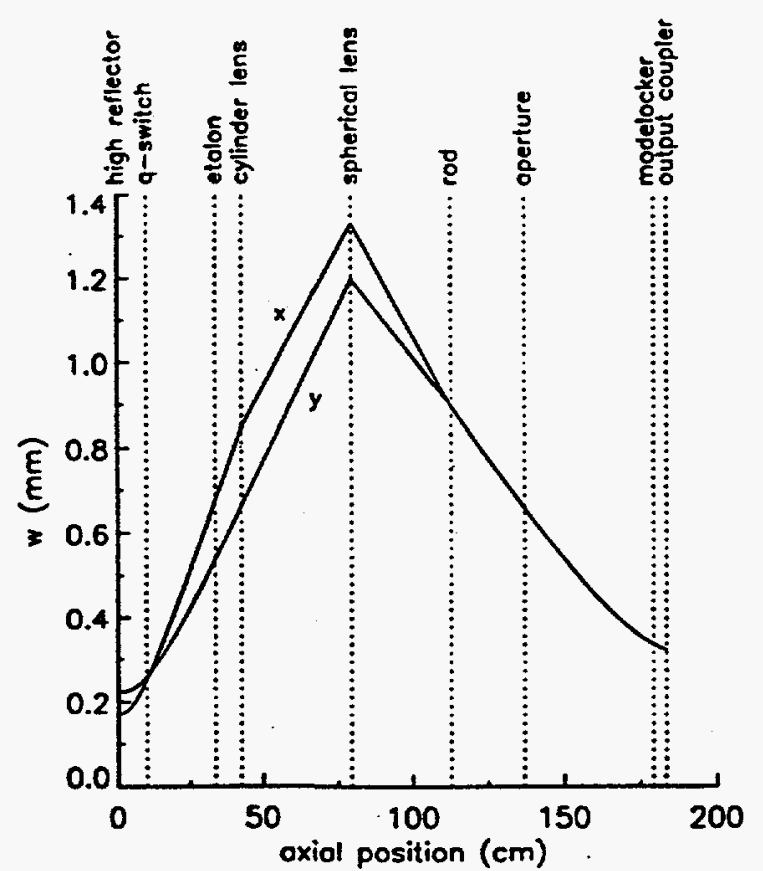

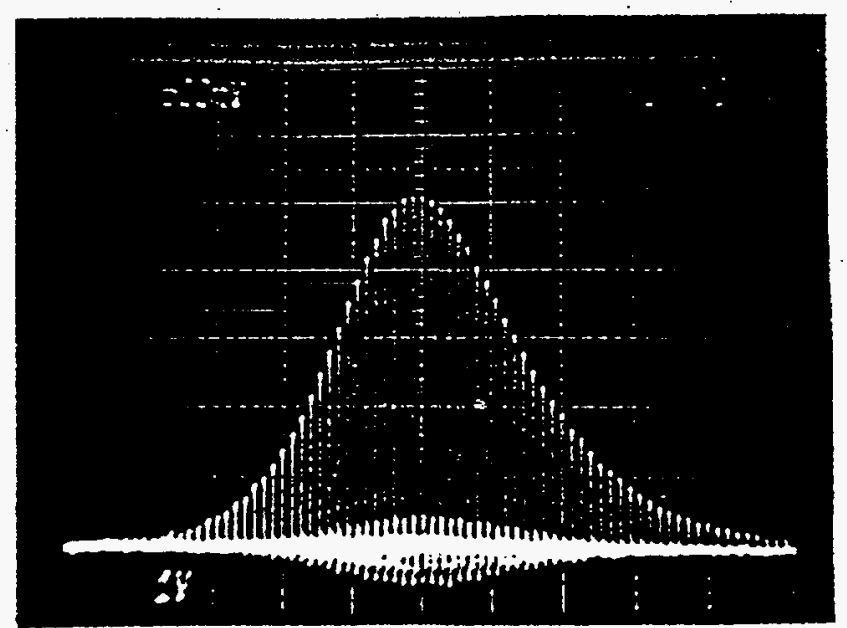

Figure 2. (a) Cavity mode profile (1/e radius, electric field) and axial positions of the components. (b) 1-GHz scope trace of the pulsed output measured with a 300-ps risetime vacuuum photodiode. $100 \mathrm{~ns} / \mathrm{div}$.

\section{The Oscillator}

The essential features of the $\mathbf{4 2 1 7}$ oscillator are summarized in Figure 2, which shows the arrangement of the components, the mode geometry and the temporal shape of the pulsed output. The active elements in this oscillator are a CW-pumped 4-mm x 10.4-cm Nd:YLF rod positioned roughly. mid-cavity, an acousto-optic quartz modelocker situated adjacent to the output coupler, and an acousto-optic quartz Q-switch located near the rear mirror. Operated CW modelocked at a pump power of $4.6 \mathrm{~kW}$ the oscillator produces $1.053-\mu \mathrm{m}$ pulses of $<70$ ps duration at a repetition rate of 75.8 $\mathrm{MHz}$ and an average power of $14 \mathrm{~W}$. When Q-switched the output consists of 3-mJ bursts of amplified modelocked pulses with the time history shown in the Figure.

The principles governing actively modelocked and Q-switched (MLQS) solid-state lasers are well known. Briefly, to achieve a stable output of amplified short pulses the oscillator must be prelased, meaning that the Q-switch loss is adjusted to allow the cavity to lase slightly and establish a well-modelocked pulse prior to Q-switching. The Q-switch loss dominates the cavity loss during this period and as a result determines the level of unsaturated gain seen by the circulating pulse when the cavity is Q-switched. The amplitude and duration of the resulting train of pulses leaked through the output coupler are therefor highly dependent on the amount of RF power delivered to the Q-switch. The pulse train shown in the Figure was produced with an RF power of $3.5 \mathrm{~W}$. The full-width-athalf-maximum (FWHM) duration of the train is $310 \mathrm{~ns}$ and the total energy is $3.1 \mathrm{~mJ}$. The energy in a modelocked pulse at the peak of the train is $120 \mu \mathrm{J}$. With a $250-\mu \mathrm{m}$ etalon installed in the cavity the FWHM modelocked pulsewidth is 90 ps. For the measurements discussed in the next section, a $1-\mathrm{mm}$ etalon was used to reduce the oscillating bandwidth to $3 \mathrm{GHz}$ and increase the modelocked pulsewidth to $150 \mathrm{ps}$.

The cavity design employs a convex output coupler $(20 \%,-120 \mathrm{~cm})$, a concave high reflector $(+200 \mathrm{~cm})$, a spherical lens $(+50 \mathrm{~cm})$ and a cylindrical lens $(+135 \mathrm{~cm})$ in a geometry based on that first 
proposed for CW modelocked Nd:YLF oscillators by Vanherzeele. ${ }^{2}$ The resulting TEM To $_{\text {mode has a }}$ large spot size in the rod that is dynamically stable relative to fluctuations in thermal lensing ${ }^{3}$ and a small spot size at the mirrors which makes the cavity "Zone I" mechanically stable in the sense described by Magni ${ }^{4}$. The mode has a waist conveniently located outside the cavity $\sim 10-\mathrm{cm}$ downstream of the output coupler.

The exact dimensions of the mode inside the cavity was not known until the spectral measurements led to a complete investigation of the cavity design. Complicating the investigation was the unknown magnitude of thermal lensing in the rod, which typically varies from rod to rod and with pump power and which is anamorphic ${ }^{5}$ (thus the need for the cylinder lens). To determine the actual mode depicted in Figure 2 it was first necessary to measure the beam size at several planes outside the oscillator-front and back- and extrapolate it to the two mirrors. Then treating the rod as a thin lens ${ }^{4}$ and using measured component spacings, the self consistent mode for the oscillator was calculated for a range of rod focal lengths to find a solution which matched the boundary conditions. This method determined the rod focal lengths to be $+700 \mathrm{~cm}$ in the vertical axis ( 1 to crystal $z$ axis) and $-350 \mathrm{~cm}$ in the horizontal axis, which is in the range of values quoted by Quantronix. The calculated mode is radially symmetric in the rod and at the output coupler with spot sizes (1/e electric-field radius) of 900 and $325 \mathrm{~mm}$ respectively. The mode size at the rear mirror is slightly elliptical with an average spot size of $200 \mu \mathrm{m}$.

The small mode size at the mirrors is a problem for MLQS operation since it results in high peak intensities inside the acousto-optic modulators. The modelocker must be positioned near a mirror to maximize mode coupling, and both modulators have wedged substrates which steer the beam out of the cavity unless positioned close enough to the mirrors. Therefor the high intensities in these devices cannot be alleviated by moving them to new locations.

\section{Spectral Measurements:}

Prior to measurement, the pulse train from the oscillator was amplified by a factor of ten in a single pass Nd:YLF rod. The amplification causes only slight modification to the temporal profile of the train and the peak intensities remain well below $1 \mathrm{GW} / \mathrm{cm}^{2}$ so that nonlinear propagation effects are not an issue. The amplified train was passed through a Pockel's cell gate to select single pulses for measurement. By varying the timing of the Q-switch trigger, pulses could be selected from different positions in the train to enable sampling the circulating cavity pulse at different stages of evolution in the Q-switch cycle.

Selected spectra obtained at progressively later sampling times in the MLQS train are organized top to bottom in Figure 3(a). The measurements were made with a Fabry Perot interferometer (FPI). The FPI was outfitted with a set of plano mirrors having matched figures of $\lambda / 200$ and reflectivities of $>98 \%$ yielding a Finesse on the order of 75 . The mirrors were configured parallel and spaced 5-mm apart for a free spectral range (FSR) of $30 \mathrm{GHz}$. The sampled pulse was expanded to a spot size of $3.5 \mathrm{~mm}$ and directed to a diffuser placed near the focal point of a $10-\mathrm{cm}$ lens situated in front of the FPI plates. The lens is not essential to the operation of the FPI but improves light collection and uniformity of illumination at the detector. The scattered radiation that passed through the FPI plates was collected by a $15-\mathrm{cm}$ focal length lens and focused onto a $C C D$ camera. The video image of the resulting ring pattern was digitized by a frame grabber and processed using software written in IDL. More details concerning the FPI configuration, resolution, and interpretation of the data are discussed in Appendix I. 

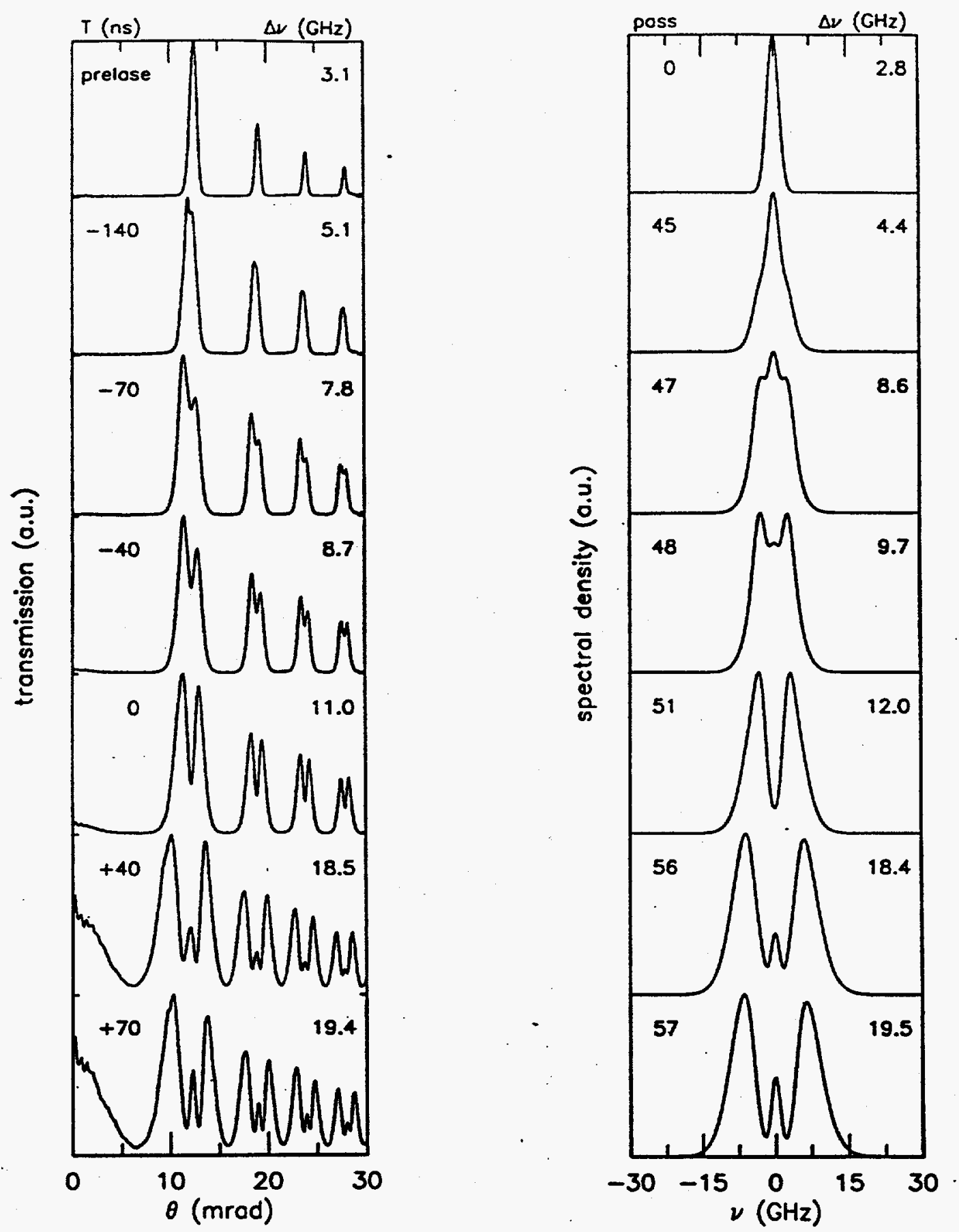

Figure 3. (a) Fabry Perot data for pulses sampled at various positions (times) in the MLQS train. Sampling times $T$ are relative to the peak of the train. (b) Numerical simulations (see text). 
The data in Figure 3(a) corresponds to single-pulse measurements with the exception of the top plot, which is a measure of the initial oscillating bandwidth prior to Q-switching. This data was obtained by integrating the nonamplified prelase signal over a $60-\mathrm{Hz}$ camera frame. The remaining data was obtained from single pulses sliced from the leading and trailing edges of the train at locations corresponding to $\sim 1 / 2$ amplitude ( $-140 \mathrm{~ns}$ ), $3 / 4$ amplitude ( \pm 70 ns), $\sim 7 / 8$ amplitude ( $\pm 40 \mathrm{~ns}$ ) and at the peak. The measurements show that the spectrum of the circulating pulse which is initially gaussian with a bandwidth of $3 \mathrm{GHz}$ is noticeably broadened by the time it reaches the half-amplitude point on the leading edge of the train. At the peak of the train the spectrum has attained a two-lobed shape and broadened to $11 \mathrm{GHz}$. On the trailing edge of the train the spectrum continues to evolve, acquiring more lobes and broadening until it eventually exceeds the 30-GHz FSR of the interferometer.

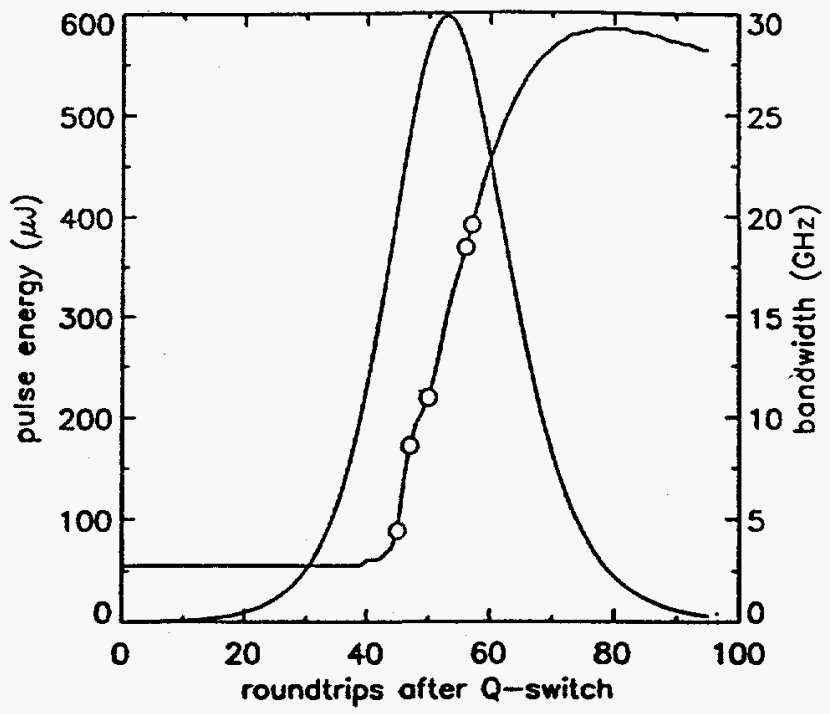

Figure 4. Calculated energy and bandwidth of the circulating modelocked pulse as a function of the number of roundrrips through the cavity since Q-switching. The circles indicate the roundtrip and bandwidth of the calculated spectra shown in Figure 3(b).

\section{Analysis}

The shape of the evolving spectrum is characteristic of that for a self-phase modulated gaussian pulse at increasing stages of chirp ${ }^{6}$. Such a pulse has the form

$$
E(t)=E_{o} e^{-\alpha t^{2}+i B(t)}, \quad B=B_{o} e^{-2 \alpha t^{2}}
$$

where $B_{0}$ is the intensity-dependent phase shift at the peak of the pulse in radians. For large $B_{0}$ the frequency spectrum becomes modulated with a number of peaks roughly equal to $B_{\sigma} / \pi$ - a fact which is not obvious but which can be verified numerically by Fourier transforming the field and taking the square of the modulus. On this basis the data appears to indicate an accumulated $B$ of $\sim \pi$ radians at the 3/4-amplitude point in the train and $\sim 2 \pi$ radians at the peak. The corresponding $\Delta B$ of $\pi$ radians over approximately five roundtrips is consistent with our estimate of .75 radians for the peak roundtrip B-integral, based on a peak circulating energy of $600 \mu \mathrm{J}$ and average spot sizes of 255 and $340 \mu \mathrm{m}$ in the $3.8-\mathrm{cm}$ long quartz modulators.

To investigate this further we developed a computer model to simulate the evolution of the circulating modelocked pulse in two dimensions ( $z$ and $t$ ) after the cavity is $Q$-switched and used it to calculate the frequency spectrum pass by pass. The model, discussed in detail in Appendix II, contains a rod with saturable gain, two passive quartz blocks with a Kerr nonlinearity for simulating propagation in the modulators, a time-dependent shutter to simulate the active modelocker, an etalon, and distributed Fresnel losses. The results of the calculations are summarized in Figures 1 and 4.

Figure 4 shows the modelocked pulse energy and FWHM bandwidth plotted continuously 
versus the number of roundtrips since Q-switching. The pulse achieves a maximum energy of $600 \mu \mathrm{J}$ after $\sim 53$ roundtrips (13.23 ns per roundtrip) with a bandwidth of $15 \mathrm{GHz}$. The maximum bandwidth achieved in the calculation is $30 \mathrm{GHz}$. Note that the energies in Figure 4 are internal and that the peak pulse energy, as well as the energy and duration of the train are consistent with the oscillator pulse of Figure 2.

The shape of the spectrum evolves as shown in Figure 1 which plots contours of spectral intensity as a function of frequency and roundtrips since Q-switching. Maximum spectral brightness occurs $\sim 10$ roundtrips before the peak of the train at roughly the 1/2-amplitude point. At the peak of the train the spectrum is clearly split into two lobes in agreement with observations. Subsequent evolution leads to three-lobed and four-lobed structure before the Q-switched gain is depleted and the oscillator drops below threshold.

The calculations and data are compared side by side in Figure 3. Essential features in the data are reproduced quite well in the model with the exception of the initial dip in the spectrum which appears narrower in the data. The calculations chosen for the Figure were those that most closely matched the data and do not in all cases correspond to roundrrips matching the quoted sampling times. For example, the spectrum and bandwidth observed at the peak of the train $(T=0)$ was best matched by the spectrum calculated for pass 51 . The two-roundtrip discrepancy is not unreasonable because the sampling time of the measurement relative to the peak is uncertain to within \pm 2 roundtrips which represents the observed jitter in the Q-switch envelope. In all cases the calculations come from roundtrips within \pm 2 roundtrips of the quoted sampling time, the exact locations indicated by the circles in Figure 4.

\section{Conclusion}

We have observed significant spectral broadening in the pulsed output of a commercial modelocked and Q-switched Nd:YLF oscillator. We identified the source to be self-phase modulation in the acousto-optic modulators and have modeled the cavity dynamics in sufficient detail to reproduce the observed spectra.

This oscillator is usually run with a thinner etalon to produce pulse lengths of $<100 \mathrm{ps}$ in which case the peak power is increased $\sim 1.5 \mathrm{x}$ and the self-phase modulation becomes much worse than that reported here. As discussed, the modulators are constrained to reside near the end mirrors and cannot simply be moved, so the fix involves a total redesign of the cavity mode. This has been done and the necessary parts are on order.

The new cavity design incorporates a planar output coupler and a convex high reflector with a 3-meter radius of curvature. The spot size of the new mode at the mirrors is $600 \mu \mathrm{m}$ and $1200 \mu \mathrm{m}$ which reduces the intensity in the modelocker and Q-switch by factors of $\sim 3$ and 20 respectively. The large mode size at the mirrors causes the resonator to reside in "Zone II" as defined by Magni ${ }^{4}$ so it will not be as mechanically stable as the current oscillator. This appears to be unavoidable. The position of the rod in the cavity has been chosen so that the mode size in the rod is dynamically stable relative to fluctuations in thermal lensing which guarantees that slight fluctuations in lamp current aren't translated into large fluctuations in laser output power. The stationary position has been calculated for a rod focal length of $+700 \mathrm{~cm}$, but the exact position will ultimately be determined as that which produces the minimum measured power fluctuation. The resulting mode size in the rod may be slightly smaller than that in the current oscillator, reducing the output power by as much as $10 \%$ but this is a consequence of optimizing the design for a rear mirror with standard radius of curvature. The 
mode waist at the output coupler is not dynamically stable which is a concern since the waist is relayed to system components and an experiment where fluctuations or changes in its size are not desirable. The focal length of the rod scales roughly with lamp current as $I^{-3 / 2}$ with $I$ typically varied from 30 to 35 amps so the maximum variation in the rod focal length is expected to be on the order of $\pm 10 \%$. In the vertical axis corresponding to the axis of positive power in the rod the dependence of waist size on lensing is predicted to be small: $\pm 2 \%$ change over the quoted range. For the horizontal axis, a 3-meter cylinder lens must be used to achieve stability about a thermal lensing value of $-300 \mathrm{~cm}$. The rod and cylinder lens form a weak telephoto with the result that the dependence of waist size on focal length will be larger in this axis: $\sim \pm 7 \%$ change over the quoted range. The current oscillator has similar sensitivity.

\section{Acknowledgments}

We wish to thank Frank Patterson, David Milam, Jim Murray and Howard Powell for several helpful discussions on this subject. Special thanks to David Eimerl for coming up with the title "Wegner's Demon".
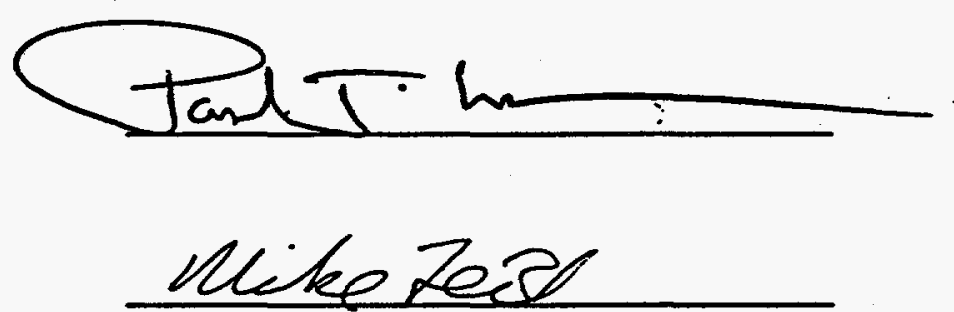


\section{Appendix I: Fabry Perot Interferometer}

The FPI was originally configured for measuring narrow linewidths in the range of 3 to 10 GHz. The transmission of this device depends strongly on angle and frequency in a nonlinear way so it is not particularly suited for characterizing complicated spectra. However bandwidths can readily be determined from the transmitted ring pattern using the relation (small angles)

$$
\frac{\Delta \nu}{\nu_{0}} \simeq \cos \theta_{1}-\cos \theta_{2} \text {. }
$$

Here $\Delta v$ is the FWHM of the power spectrum and the angles $\theta_{1,2}$ delimit the FWHM of the transmission peak. The effective focal length of the collecting lens is $f$ so if $r$ is the radius at the CCD chip as measured from the digitized image then $\theta$ is just $\arctan (r / f)$. Thus accurate measurement of bandwidth depends on accurate measurement of radii at the $C C D$, and knowledge of $f$. Accuracy of the bandwidth calculation can be verified if there are several rings in the image on which it can be repeated.

The resolution with which $r$ can be measured is limited by the $25-\mu \mathrm{m}$ pixel size of the $C C D$ and the diffraction limited spot size of the collecting lens. The F-number of the lens was fixed at ten to produce a diffraction limited spot size of 1.1 pixels at $1.053 \mu \mathrm{m}$. The lowest acceptable limit for $\Delta r E r_{2}-r_{1}$ given a linewidth of $5 \mathrm{GHz}$ was set at three pixels and the number of rings inside the 7x10-mm aperture of the chip that is accessed by the frame grabber was set at three to four. Since $\Delta \theta \equiv \theta_{2}-\theta_{1}$ is inversely proportional to $\sin \theta$ it is advantageous to generate the four transmission maxima at fairly small angles and spread them out over the chip with a lens of suitable focal length. Therefor a FSR of $30 \mathrm{GHz}$ was chosen to produce four rings inside an angle of $27 \mathrm{mrad}$ and a $15-\mathrm{cm}$ - lens used to focus the rings on the chip within a radius of $4 \mathrm{~mm}$. An IDL code was written which determines the center of the ring pattern by interactive selection of three points on any given ring, performs a radial average of the image, locates the peaks of the rings and determines the linewidth. Partial rings in the corners of the image are also analyzed. Using this method, linewidths with values within $\pm 5 \%$ are obtained from as many as four rings.

A final comment concerns the effective focal length of the collecting lens. In practice the lens is positioned to achieve the sharpest image on the $\mathrm{CCD}$ and the focal length is not well known. For this application a plano-convex lens with known radius of curvature and center thickness was used. These parameters were used in a simple thick-lens ray-trace code written in IDL to determine the location of the best image plane for the angular ranges considered above. The position of this plane was then verified by direct measurement. The code calculates the transverse position of the focus for a given angle of incidence which determines the effective focal length.

\section{Appendix II: Numerical Model}

Referring to Figure 2, we wish to follow an initially steady-state modelocked pulse around the cavity starting at the point in time when the power to the Q-switch is turned off. Suitable initial conditions are a well-formed 150-ps gaussian pulse arbitrarily situated at the output coupler and a uniformly pumped rod with a small signal gain to be determined. Starting at the output coupler the pulse first passes through the modelocker where it is modulated in amplitude and undergoes self phase modulation, then the rod where it is amplified, and on through the lenses, etalon and Q-switch before being reflected by the rear high reflector and passed back through the components in reverse order to 
output coupler. The process is repeated until the gain in the rod is depleted.

In the simplest description of this process we neglect both the spatial dependence of the field as well as second-order time effects. The first approximation is moderately justified by the fact that the size of the cavity mode does not vary significantly over the length of the individual components. The second is based on initial analysis which showed group velocity dispersion to have little effect on the propagation of the pulse through the $\mathbf{3 0}$ meters or so of material traversed in the calculation. The reduced problem involves moving a plane-wave approximation of the field with appropriate scaling from component to component around the cavity. The field is defined as usual in terms of a slowlyvarying complex amplitude $A$

$$
E(z, t)=\frac{1}{2} A(z, t) e^{i\left(k_{0} z-\omega_{0} t\right)}+c . c, \quad k_{0}=\frac{n \omega_{0}}{c}
$$

After transforming to a local time coordinate $\tau=t-2 / v_{\mathrm{g}}$ the evolution of the complex field amplitude is tracked through the various components as described below. To move between components the amplitude is multiplied by the scale factor

$$
S=\frac{w_{i}}{w_{f}} \sqrt{\frac{n_{i}}{n_{f}}\left(1-R_{i}\right)\left(1-R_{f}\right)}
$$

where $n_{i f}$ is the refractive index, $w_{L f}$ is the spot size and $R_{L f}$ is the Fresnel loss in the initial and final components. The value used in the code for the refractive index of both fused silica and YLF is 1.45. The spot sizes in the Q-switch, modelocker and rod as determined from Figure 2 are 255 rm, 340 pm and $900 \mu \mathrm{m}$. The reflectivity of all surfaces in the cavity is estimated at $0.25 \%$ except for the output coupler and high reflector which are assigned values of $80 \%$ and $99.5 \%$ respectively.

In the modelocker there is both time-varying transmission and nonlinear refractive index to consider. The modulating property of the device is simulated with a time-dependent shutter positioned in the vicinity of the substrate. The shutter has the following amplitude-transmission function ${ }^{7}$

$$
T_{m}(t)=\cos \left(\theta_{m} \sin \left(2 \pi u_{m} t\right)\right)
$$

where parameters appropriate for the Quantronix modelocker are a modulation frequency $V_{m}$ of 37.8 $\mathrm{MHz}$, and a modulation depth $\theta_{m}$ of $\sim 0.5$. In reality, the role of amplitude modulation in the model is not that critical. The modelocker does most of its work during the prelase period so that by the time the cavity is Q-switched the circulating pulse is well formed and arrives at the modelocker after each roundtrip coincident in time with the peak of the transmission function. Compared to the short duration of the pulse the transmission function is very broad, exceeding $98.5 \%$ for over 3 ns and so has only a minor effect on the shape of the pulse during the 70 or so roundrips of the Q-switch cycle. Simulations with and without the shutter show it may be omitted without affecting the result.

To simulate the nonlinear propagation in the modelocker and the Q-switch the model incorporates two $3.85-\mathrm{cm}$ long passive blocks of fused silica with an intensity-dependent refractive index. With the approximations discussed previously, the field amplitude can be shown to evolve in the blocks according to

$$
\frac{\partial}{\partial z} A(z, \tau)=i \frac{k_{0}}{n} \gamma I(z, \tau) A(z, \tau), \quad I=\frac{1}{2} n \varepsilon_{o} c|A|^{2}
$$


where $\gamma=2.75 \times 10^{-7} \mathrm{~cm}^{2} / \mathrm{GW}$ is the nonlinear coefficient of the material. Numerically the blocks are divided into several increments $d z$, and the field assigned a phase shift after each step of

$$
B(z, \tau)=\frac{k_{0}}{n} \gamma I(z, \tau) d z .
$$

The number of increments is chosen to keep the incremental phase shift small and a choice of two to four steps for these calculations resulted in peak phase shifts of less than .1 radians/step.

The propagation of the pulse through the laser rod is described using the Frantz-Nodvik solutions applicable to saturable, homogeneously broadened gain media ${ }^{8}$

$$
\begin{aligned}
& A(L, \tau)=A(0, \tau) /\left[1-\left(1-e^{-\sigma \int_{0}^{L} \Delta_{i}\left(z^{\prime}\right) d z^{\prime}}\right) e^{-\frac{2 \sigma}{h \nu} \int_{-\infty}^{\tau} I_{0}\left(\tau^{\prime}\right) d \tau^{\prime}}\right]^{1 / 2} \\
& \Delta_{f}(z)=\Delta_{i}(z) e^{-\sigma \int_{0}^{z} \Delta_{i}\left(z^{\prime}\right) d z^{\prime}} /\left[e^{\frac{2 \sigma}{h \nu} \int_{-\infty}^{\infty} I_{0}\left(\tau^{\prime}\right) d \tau^{\prime}}+e^{-\sigma \int_{0}^{z} \Delta_{i}\left(z^{\prime}\right) d z^{\prime}}-1\right] .
\end{aligned}
$$

These equations are solved for each pass through the rod. $I_{0}$ is the pulse intensity at the rod input, $\Delta_{i f}$ is the $z$-dependent population inversion density in the rod before and after each pass, $\sigma$ is the stimulated emission cross section $\left(1.2 \times 10^{-19} \mathrm{~cm}^{2}\right)$ and $L$ is the length of the $\operatorname{rod}(10.4 \mathrm{~cm})$. At the start of the simulation, $\Delta_{l}$ is assumed to be uniform throughout the rod with some steady-state value determined by the Q-switch loss. For each pass of the pulse through the rod, the final z-dependent population inversion density $\Delta_{f}$ is calculated, rotated in $z$, and stored for use as the initial inversion density $\Delta_{i}$ on the next pass. Implicit in this method is the assumption that the pumping rate is slow and does not contribute significantly to the inversion density during the sub-microsecond duration of the Q-switch cycle. The assumption is reasonable since the pumping times are more on the order of $600 \mu \mathrm{s}$ which is the time observed for the oscillator to reachieve threshold once the gain is depleted by Q-switching. To adequately evaluate the integrals inside the exponentials the inversion density is specified at $\mathbf{5 1 2}$ points along the rod. The appropriate initial value was determined empirically to achieve an output pulse train of the correct energy and duration. The optimum value corresponds to an initial small signal gain of $.022 \mathrm{~cm}^{-1}$.

The final component to consider is the etalon. It has the following complex frequencydependent transmission

$$
T_{e}(\omega)=\frac{1-R}{1-R e^{-i \delta(\omega)}}, \quad \delta(\omega)=\frac{2 \pi d}{c} \omega
$$

where $R$ is the reflectivity $(.035), d$ the thickness $(.1 \mathrm{~cm})$ and $n$ the refractive index (1.45). This transmission function is applied to the Fourier transform of the field amplitude $A$. Note then that the variable $\omega$ here represents a frequency shift relative to the carrier frequency $\omega_{0}$ about which the transmission peak of the etalon is assumed to be centered. Before applying this transmission function the phase is first extracted and modified numerically to remove the linear frequency component. This eliminates an annoying time shift of the pulse that normally occurs as a result of finite transit time across the etalon. The phase shift calculated for the etalon is shown before and after modification in Figure 5. 
The initial gaussian pulse amplitude $A(0, \tau)$ is constructed on a 1024-pt grid. A peak electric-field amplitude of $2.6 \times 10^{4} \mathrm{~V} / \mathrm{cm}$ and a $1 / \mathrm{e}$ halfwidth of $127 \mathrm{ps}$ were chosen to correspond to a steady state circulating prelase pulse with a FWHM $\Delta \tau$ of 150 ps and an energy of 250 $\mathrm{nJ}$ in a spotsize of $340 \mu \mathrm{m}$. Fast Fourier transforms are used to extract the pulse spectra after each roundtrip of the cavity (as well as for propagation through the etalon) so the width of the grid $\tau_{g}$ is chosen so that the initial pulse is resolved equally in both time and frequency. The requirement

$$
\frac{\Delta \tau}{\tau_{g}}=\sqrt{2 \ln 2 / n_{g} \pi}
$$

where $n_{g}$ is the number of grid points results in a grid width of $7.2 \mathrm{~ns}$ and a temporal resolution of $7.1 \mathrm{ps}$. The corresponding grid in the frequency domain spans $140 \mathrm{GHz}$ and has a resolution of $135 \mathrm{MHz}$.

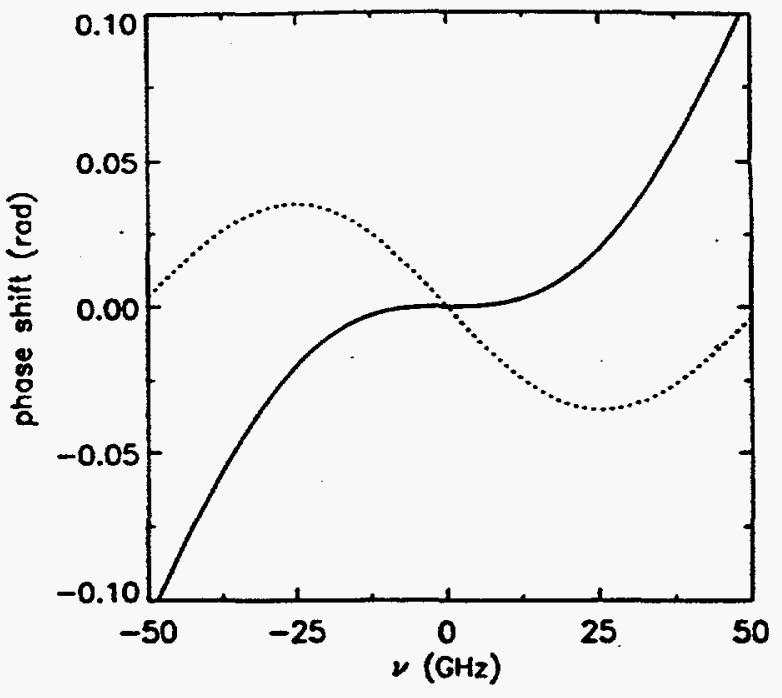

Figure 5. Plot showing the phase of the etalon transmission function before ( $(.$.$) and after( \longrightarrow$ subtraction of the linear frequency term.

\section{References}

1. D.J. Kuizenga, "Short-Pulse oscillator development for the Nd:Glass Laser Fusion Systems, " IEEE J. Quantum Electron. QE-17, 1694-1708 (1981).

2. H. Vanherzeele, "Thermal lensing measurement and compensation in a continuous-wave modelocked Nd:YLF laser," Opt. Lett. 13, 369-371 (1988).

3. J.P. Lortscher, J. Steffen and G. Herziger, "Dynamic stable resonators: a design procedure," Opt. Quantum Electron. 7, 505-514 (1975).

4. V. Magni, "Multielement stable resonators containing a variable lens," J. Opt. Soc. Am. A 4, 1962 1969 (1987).

5. J.E.:Murray, "Pulsed Gain and Thermal Lensing of Nd:LiYF," IEEE J. Quantum Electron. QE-19, 488-491 (1983).

6. see for example A. E. Siegman, Lasers (University Science Books, Mill Valley Cal., 1986), pp. 382-386.

7. R. Adler, "Interaction between light and sound," IEEE Spectrum 4, $42-54$ (1967).

8. L.M. Frantz and J.S. Nodvik, "Theory of Pulse Propagation in a Laser Amplifier," J. Appl. Phys. 34, 2346-2349 (1963). 